\title{
Molecular characterization and new genotypes of Enterocytozoon bieneusi in pet chipmunks (Eutamias asiaticus) in Sichuan province, China
}

Lei Deng ${ }^{1+} \mathbb{B}$, Wei $\mathrm{Li}^{1+}$, Zhijun Zhong ${ }^{1+}$, Yijun Chai ${ }^{1}$, Leli Yang ${ }^{1}$, Hang Zheng ${ }^{1}$, Wuyou Wang ${ }^{1}$, Hualin Fu ${ }^{1}$, Min He${ }^{1}$,

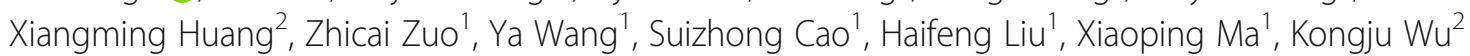
and Guangneng Peng ${ }^{1 *}$

\begin{abstract}
Background: Enterocytozoon bieneusi, the most commonly identified microsporidian species in humans, is also identified in livestock, birds, rodents, reptiles, companion animals, even wastewater. However, there is no information available on occurrence of E. bieneusi in pet chipmunks. The aim of the present study was to determine the genotypes, molecular characterization of E. bieneusi in pet chipmunks, and assess the zoonotic potential.

Results: A total of 279 fecal specimens were collected from chipmunks from seven pet shops and one breeding facility in Sichuan province, China. The prevalence for E. bieneusi was 17.6\% (49/279) based on nested PCR targeting the internal transcribed spacer (ITS) region. The prevalence of E. bieneusi in chipmunks $<90$ days of age was significantly higher than that in older chipmunks; however, differences among different sources and between genders were not significant. Eight genotypes of E. bieneusi were identified, including four known genotypes ( $D$, Nig7, CHG9, and CHY1) and four novel genotypes (SCC-1 to 4). Phylogenetic analysis classified these genotypes into four distinct groups as follows: genotypes D and CHG9 clustered into group 1 of zoonotic potential; genotypes Nig7 and CHY1 clustered into group 6 and a new group, respectively; the four novel genotypes (SCC-1 to 4) formed a separate group named group 10.

Conclusions: To the best of our knowledge, this is the first study reporting the prevalence and genotypes of E. bieneusi in pet chipmunks in China. Genotypes D and Nig7, found in chipmunks in this study, have also been previously identified in humans, which suggests that chipmunks might play a role in the transmission of this pathogen to humans.
\end{abstract}

Keywords: Enterocytozoon bieneusi, Chipmunks, Prevalence, Genotype, Zoonotic potential

\section{Background}

Microsporidia comprise a large and diverse group of intracellular eukaryotes that infects invertebrate and vertebrate hosts worldwide; to date, it consists of approximately 1300 species in 160 genera [1]. Enterocytozoon bieneusi is the most frequently detected species of

\footnotetext{
* Correspondence: pgn.sicau@163.com

${ }^{\dagger}$ Equal contributors

${ }^{1}$ The Key Laboratory of Animal Disease and Human Health of Sichuan Province, College of Veterinary Medicine, Sichuan Agricultural University, Province, Chengdu 611130, Sichuan, China

Full list of author information is available at the end of the article
}

microsporidia and is responsible for greater than $90 \%$ of human microsporidiosis cases $[2,3]$. E. bieneusi usually causes self-limiting diarrhea and malabsortion in healthy individuals [4]. However, it can cause life-threatening diarrhea in individuals with deficient immune systems, such as AIDS patients and transplant recipients [5].

Genotypes of E. bieneusi have been determined based on sequence analysis of the internal transcribed spacer (ITS) region of ribosomal RNA (rRNA) [1]. To date, more than 240 genotypes of E. bieneusi have been identified in humans and animals $[6,7]$. The ITS genotypes

(c) The Author(s). 2018 Open Access This article is distributed under the terms of the Creative Commons Attribution 4.0 International License (http://creativecommons.org/licenses/by/4.0/), which permits unrestricted use, distribution, and 
of $E$. bieneusi have been divided into nine different groups based on phylogenetic analyses [8]. Group 1, considered zoonotic, is frequently identified in humans and animals [9]. In contrast, the remaining groups (groups 2-9) are considered host-adapted groups and have no significant public health importance $[10,11]$.

In China, E. bieneusi has been reported in humans, livestock, companion animals, and wastewater, and some genotypes of this species have been identified in both humans and animals $[10,12,13]$. Chipmunks (Eutamias asiaticus) have become popular in China as companion animals. However, there is no epidemiological information regarding the prevalence of $E$. bieneusi in chipmunks. The aim of this study was to determine the prevalence and genotypes of E. bieneusi in chipmunks, as well as to assess the zoonotic potential of this organism as it relates to pet chipmunks and humans.

\section{Method}

\section{Collection of specimens}

A total of 279 fecal specimens were obtained from chipmunks between March 2016 and April 2017 from seven pet shops and one breeding facility in Sichuan province, southwestern China (Table 1). The fecal samples were collected from the bottom of cages after defecation and then immediately placed into individual $30-\mathrm{mL}$ sterile containers. All the fecal samples were taken to the laboratory in a cooler with ice packs within $24 \mathrm{~h}$. All the chipmunks were in apparently good health at the time sampling and the age, gender and source was also recorded at the same time.

\section{DNA extraction and PCR amplification}

All the fecal specimens were washed three times by centrifugation at $1500 \mathrm{~g}$ for $10 \mathrm{~min}$ with distilled water. Genomic DNA was extracted from approximately $200 \mathrm{mg}$ of each processed fecal specimen using the E.Z. N.A.R ${ }^{\circ}$ Stool DNA kit (Omega Biotek Inc., Norcross,
USA) according to the manufacturer's recommended instructions. The extracted DNA was stored at $-20{ }^{\circ} \mathrm{C}$ until molecular analysis.

E. bieneusi was determined by nested PCR amplification of a 392-bp fragment, containing the entire ITS (243 bp) and the portions of the flanking large and small subunits of the rRNA gene. The primers and cycling conditions in nested PCR were used as previously described by Sulaiman et al. [14]. TaKaRa Taq ${ }^{\text {Tw }}$ DNA Polymerase (TaKaRa Bio, Otsu, Japan) was used for PCR amplifications. A negative control with no DNA added was included in all the PCR tests. The secondary PCR products were examined by agarose gel electrophoresis and visualized after ethidium bromide staining.

\section{Sequence and phylogenetic analyses}

All amplified products were sequenced by Life Technologies (Guangzhou, China) using a BigDye Terminator v3.1 cycle sequencing kit on an ABI 3730 DNA Analyzer (Applied Biosystems, Foster City, CA). Nucleotide sequence accuracy was confirmed by sequencing of two separate PCR products. The obtained sequences in this study were aligned with reference sequences downloaded from GenBank using the program ClustalX 2.0 (http://www.clustal.org/) to determine the genotypes. The genotypes from this study were compared with previously published $E$. bieneusi ITS genotypes using a neighbor-joining analysis of the aligned $E$. bieneusi sequences implemented in the program Mega 6 (http:// www.megasoftware.net/), and a bootstrap analysis with 1000 replicates was performed to assess the robustness of clusters.

\section{Statistical analysis}

Differences in infection rates were compared using the chi-square test and difference was considered significant when $p<0.05$. The analysis was done using SPSS version 17.0 (SPSS Inc., Chicago, IL USA).

Table 1 Prevalence and genotypes of E. bieneusi in pet chipmunks from different sources in Southwestern China

\begin{tabular}{llll}
\hline Source & No. of animals & No. of positive (\%) & Genotypes (n) \\
\hline Pet shop1 & 24 & $7(29.2 \%)$ & D (2); CHG9 (1); SCC-1 (4) \\
Pet shop2 & 30 & $4(13.3 \%)$ & SCC-1 (4) \\
Pet shop3 & 28 & $6(21.4 \%)$ & D (1); CHY1 (2); SCC-3 (3) \\
Pet shop4 & 14 & $2(14.3 \%)$ & SCC-3 (2) \\
Pet shop5 & 19 & $6(31.6 \%)$ & Nig7 (2); SCC-2 (4) \\
Pet shop6 & 35 & $5(17.1 \%)$ & CHG9 (1); SCC-2 (5) \\
Pet shop7 & 26 & $13(12.6 \%)$ & SCC-1 (5) \\
Breeding facility & 103 & $49(17.6 \%)$ & D (3); Nig 7 (2); CHY1 (3); SCC-1 (4); SCC-4 (1) \\
Total & 279 & D (6); Nig 7 (4); CHG9 (2); CHY1 (5); SCC-1 (17); \\
& & SCC-2 (9); SCC-3 (5); SCC-4 (1) \\
\hline
\end{tabular}


Table 2 Prevalence and genotypes of E. bieneusi in pet chipmunks by age and gender

\begin{tabular}{llll}
\hline Group & No. of animals & No. of positive & Infection rate \\
\hline Age & & & \\
$<90$ days & 146 & 35 & $24.0 \%$ \\
$90-270$ days & 53 & 8 & $15.1 \%$ \\
$>270$ days & 80 & 6 & $7.5 \%$ \\
Sex & & & \\
Male & 125 & 19 & $15.2 \%$ \\
Female & 154 & 30 & $19.5 \%$ \\
\hline
\end{tabular}

\section{Results}

\section{Prevalence of $E$. bieneusi in chipmunks}

Of the 279 fecal samples examined for E. bieneusi by PCR amplification of the ITS gene, 49 (17.6\%) were positive. All tested pet shops have E. bieneusi infection, and infection rates ranged from 12.6 to $31.6 \%$ (Table 1). The highest infection rate was observed in pet shop $5(31.6 \%, 6 / 19)$, and it was apparently higher than that in other pet shops, but the difference was not significant $(P>0.05)$. Infection rates of E. bieneusi in chipmunks of different ages and sexes are shown in Table 2. The highest prevalence of E. bieneusi was observed in chipmunks < 90 days of age $(24 \%, 35 /$ 146), followed by that in 90-270-day-old chipmunks $(15.1 \%, 8 / 53)$, and in $>270$-day-old chipmunks $(7.5 \%$, $6 / 80)$; the differences among these groups was significant $(P<0.05)$. The prevalence of $E$. bieneusi was also higher in females (19.5\%) than in males (15.2\%), but the difference was not significant $(P>0.05)$.

\section{Genotype distribution and genetic characterization of $E$. bieneusi in chipmunks}

Eight genotypes were identified in the present study by sequence analysis of the ITS gene of $49 \mathrm{E}$. bieneusi-positive fecal specimens; these genotypes included four known genotypes (D, Nig7, CHG9, and CHY1) and four novel genotypes named SCC-1 to SCC-4. Among these genotypes, genotype SCC-1 was the most prevalent (34. $7 \%, 17 / 49)$, followed by SCC- $2(18.4 \%, 9 / 49)$, and D (12. $2 \%, 6 / 49)$. Five genotypes were identified in the breeding facility, including two known zoonotic genotypes, D and Nig7.

A high degree of genetic polymorphism was observed among the novel genotypes. The base variation of the novel genotypes within the $243 \mathrm{bp}$ of the ITS sequence is presented in Fig. 1.

\section{Phylogenetic analysis}

Phylogenetic analysis, using the neighbor-joining method based on the ITS sequences of E. bieneusi, showed that all positive samples found in the present study belonged to four groups. Genotypes D and CHG9 clustered into group 1 and were further classified into subgroups $1 \mathrm{a}$ and $1 \mathrm{f}$, respectively (Fig. 2). Genotype Nig7 clustered into group 6 , and genotype CHY1 was classified as a new cluster. The four novel genotypes (SCC-1 to 4) were separated into a new group, named group 10.

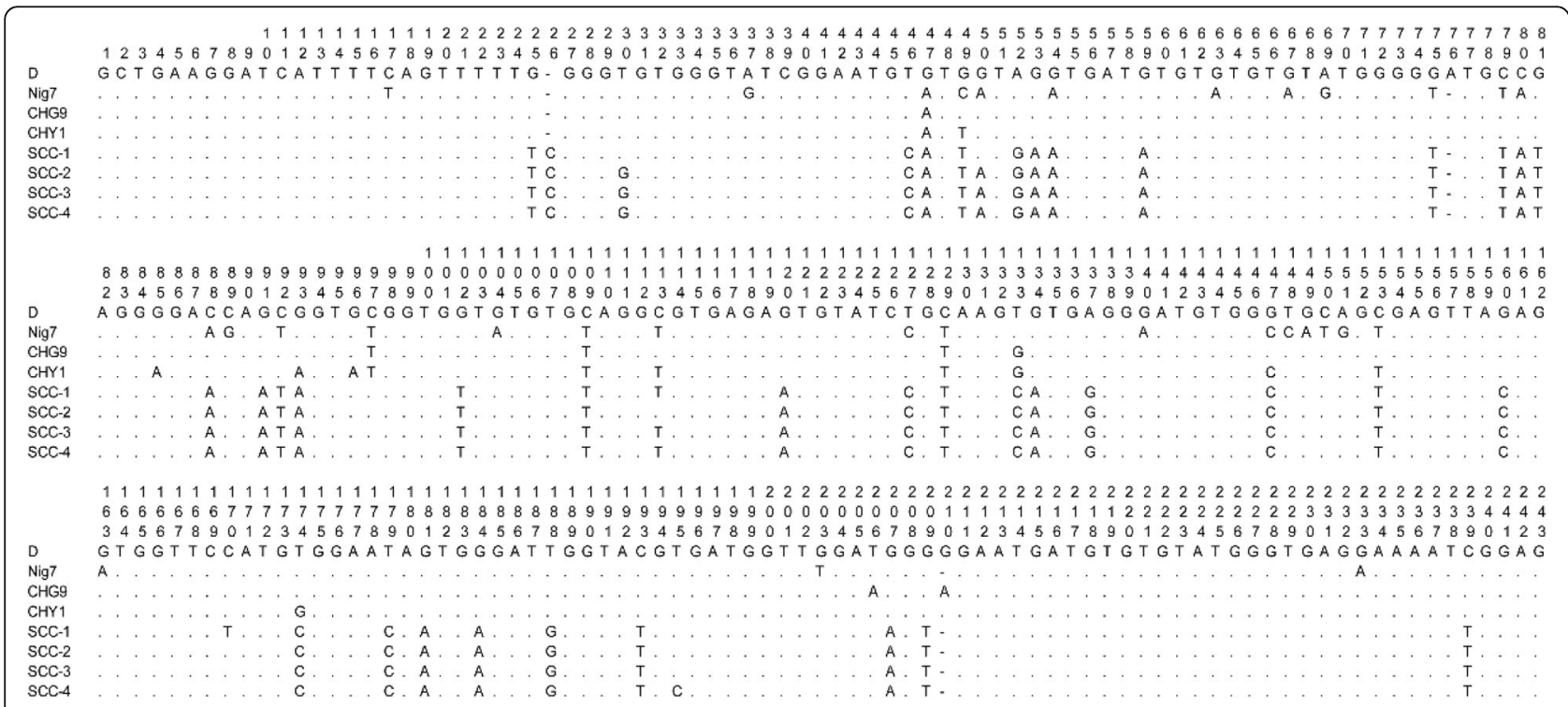

Fig. 1 Sequence variation in the ITS region of the rRNA gene of Enterocytozoon bieneusi isolates from pet chipmunks. The ITS sequences of four known genotypes (D, Nig7, CHG9, and CHY1) and the four novel genotypes (SCC-1 to 4), identified in this study, were aligned with each other. The dots and transverse lines indicate base identities and deletions, respectively, relative to the ITS sequence of genotype D 


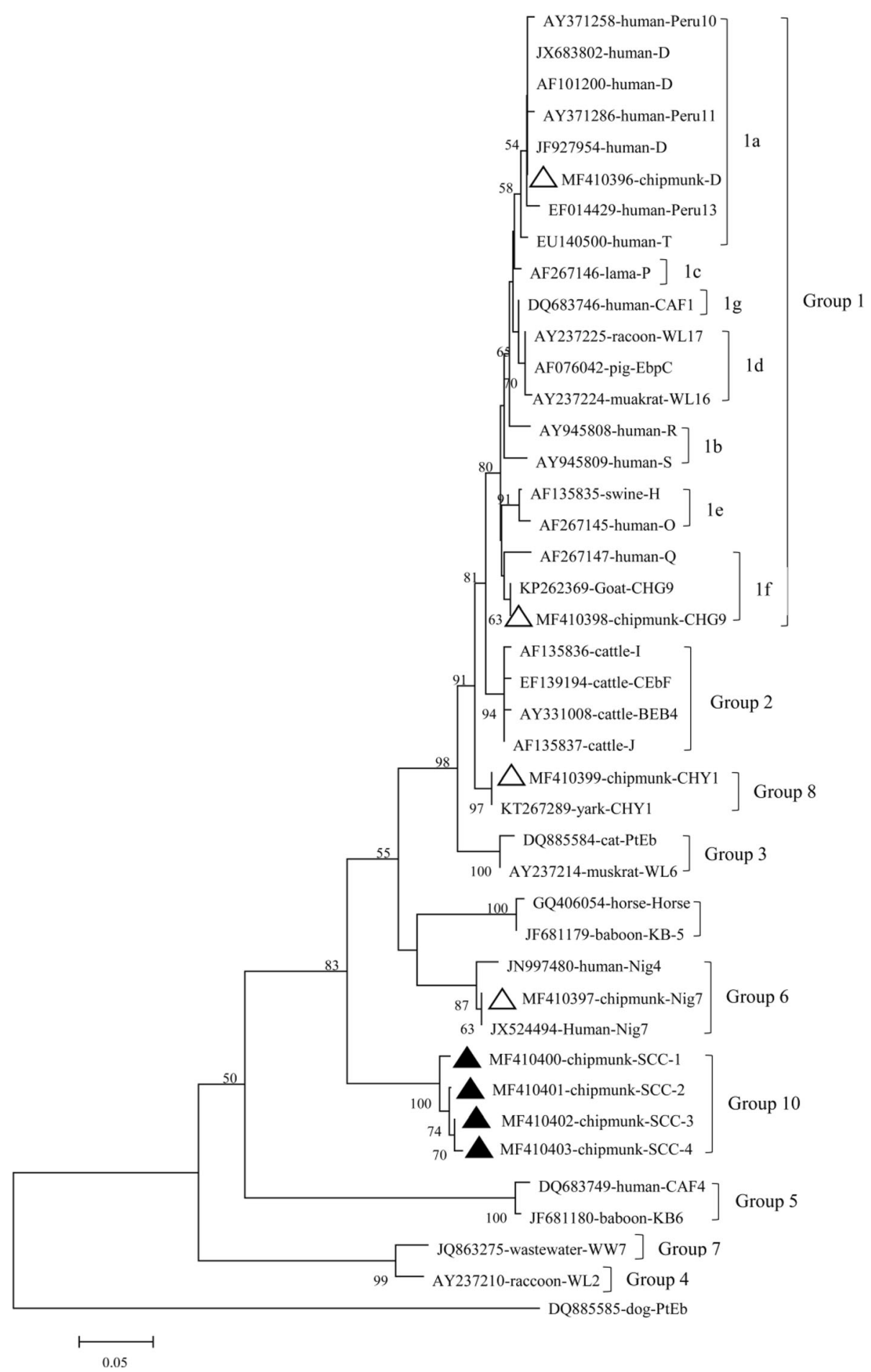

Fig. 2 Phylogenetic relationship of Enterocytozoon bieneusi genotypes identified in this study and other genotypes previously deposited in GenBank as inferred by a neighbor-joining analysis of ITS sequences based on genetic distances calculated by the Kimura 2-parameter model. The number on the branches are percent bootstrapping values from 1000 replicates, with values of more than 50\% shown in the tree. Each sequence is identified by its accession number, host origin, and genotype. Genotypes with open triangles and black triangles are known and novel genotypes identified in this study, respectively 


\section{Discussion}

In this study, we determined for the first time, the prevalence $(17.6 \%)$ of $E$. bieneusi in chipmunks in China. At present, only two studies have described the prevalence of $E$. bieneusi in pet rodents in China; specifically, 3.6\% of 140 pet chinchillas in Henan province and $16.7 \%$ of 144 pet red-bellied tree squirrels in Sichuan province were found to harbor this pathogen $[6,15]$. However, there have been many reports of wild rodents with $E$. bieneusi infection, worldwide; a high prevalence (38.9\%) was found in wild small rodents in Poland [16], 26.8\% incidence was identified in wild rodents in New York [17], and $10.7 \%$ of wild mice were infected in the Czech Republic [18], whereas the lowest prevalence (1.0\%) was identified in wild mice in Slovakia [19]. Differences between these studies could be explained by different geographical regions, sample sizes, management methods, age, and seasonal variations.

We also identified eight genotypes by analyzing the ITS sequences; these genotypes included four known genotypes (D, Nig7, CHG7, and CHY1) and four novel genotypes (SCC-1 to 4). In previous studies, eight genotypes (D, C, H, EbpA, Peru 8, S6, CZ3, and PigEBITS5) were identified in wild mice in a hybrid zone across the Czech Republic-Germany border [18], 12 (D, gorilla 1, and WR1-10) were found in wild rodents in Poland [16], two (D and BEB6) in pet chinchillas in China [15], and five (D, EbpC, SC02, CE01, and CE02) in red-bellied tree squirrels in China [6]. Together, these results show that genotype D has widespread geographical distribution and is very common in rodents. In addition, genotype $\mathrm{D}$ has also been identified in various hosts in China, such as humans, non-human primates, cattle, pigs, dogs, foxes, cats, goats, horses, and sheep, as well as in wastewater [5, 9, 11, 12, 20-22]. Genotype D has already been considered as a zoonotic genotype of public health significance. Genotype Nig7 was originally reported in HIV-infected patients in Nigeria [23], and genotypes CHG9 and CHY1 have been previously identified in goats and yaks in China [21, 24], respectively. These genotypes were all identified for the first time in chipmunks in China, suggesting that chipmunks play a potential role in the transmission of E. bieneusi to humans and other animals, acting as a reservoir host.

Genetic relationships between the E. bieneusi genotypes obtained in this study and known strains were identified based on phylogenetic analysis. The two known genotypes D and CHG9 belonged to group 1, which is composed of genotypes almost exclusively from humans [25-28]; this result suggested the potential for zoonotic transmission and indicates the public health significance of these genotypes [29]. Genotype CHY1 was classified as being a member of a new cluster, which contains genotypes from different animals, such as
CHB1 from bears and CHK1-2 from kangaroos [10]. Genotype Nig7 clustered into group 6; this group was first identified in wastewater and has been determined to be capable of infecting a broad range of hosts including humans, non-human primates, horses, and squirrels $[5,30,31]$. The remaining four novel genotypes (SCC-1 to SCC-4) were clustered into a separate cluster, which is divergent from other known genotype groups, and appears to be specific to chipmunks; we named this Group 10. However, it remains difficult to determine if the novel genotypes have the ability to cause human microsporidiosis and have a broader host spectrum; future studies should be aimed at investigating the potential of the genotypes in these groups to cause disease in humans and other animals.

\section{Conclusions}

This is the first report of the prevalence and genotypes of $E$. bieneusi in chipmunks from China; this study also identified a new chipmunk-specific group, named group 10. The detection of two known genotypes (D and Nig7) that are also common to humans and the fact that the genotype CHG9 belonged to group 1 suggests that chipmunks infected with $E$. bieneusi might pose a threat as a route of transmission to humans.

\section{Abbreviations}

E. bieneusi: Enterocytozoon bieneusi; ITS: internal transcribed spacer; rRNA: ribosomal RNA

\section{Acknowledgements \\ We thank Qicheng Xiao and Run Luo for collecting samples.}

\section{Funding}

The study was financially supported by the Chengdu Giant Panda Breeding Research Foundation (CPF2014-10; CPF2014-14; CPF2015-4). The funders contributed to the study design and data collection.

\section{Availability of data and materials}

Representatives of all the nucleotide sequences generated in this study have been deposited in GenBank (https://www.ncbi.nlm.nih.gov/) under the accession numbers MF410396-MF410403. The datasets used and/or analysed during the current study are available from the corresponding author on reasonable request.

\section{Authors' contributions}

Experiments were conceived and designed by GP and LD. WL, ZZ1, YC, LY and $\mathrm{HZ}$ collected samples. Experiments were performed by LD, XH, XM, ZZ2, $\mathrm{HF}, \mathrm{MH}, \mathrm{WW}, \mathrm{YW}$ and SC, and the data were analyzed by LD, KW and $\mathrm{HL}$. The manuscript was written by LD. All authors have read and approved the submitted version of this manuscript. ZZ1 would correspond to the author furthest up on the author list.

\section{Ethics approval and consent to participate}

The present study protocol was reviewed and approved by the Research Ethics Committee and the Animal Ethical Committee of Sichuan Agricultural University. Permission was obtained from the shop owners or managers before the fecal specimens were collected.

Competing interests

The authors declare that they have no competing interests. 


\section{Publisher's Note}

Springer Nature remains neutral with regard to jurisdictional claims in published maps and institutional affiliations.

\section{Author details}

'The Key Laboratory of Animal Disease and Human Health of Sichuan Province, College of Veterinary Medicine, Sichuan Agricultural University, Province, Chengdu 611130, Sichuan, China. ${ }^{2}$ Chengdu Giant Panda Breeding Research Base, Province, Chengdu 625001, Sichuan, China.

\section{Received: 9 August 2017 Accepted: 5 April 2018}

Published online: 18 April 2018

\section{References}

1. Huang J, Zhang Z, Yang Y, Wang R, Zhao J, Jian F, et al. New genotypes of Enterocytozoon bieneusi isolated from Sika deer and red deer in China. Front Microbiol. 2017;8:879.

2. Tian GR, Zhao GH, Du SZ HXF, Wang HB, Zhang $L X$, et al. First report of Enterocytozoon bieneusi from giant pandas (Ailuropoda melanoleuca) and red pandas (Ailurus fulgens) in China. Infect Genet Evol. 2015;34:32-5.

3. Ye J, Ji Y, Xu J, Ma K, Yang X. Zoonotic Enterocytozoon bieneusi in raw wastewater in Zhengzhou, China. Folia Parasitol. 2017;64

4. Li W, Cama V, Feng Y, Gilman RH, Bern C, Zhang X, et al. Population genetic analysis of Enterocytozoon bieneusi in humans. Inter J Parasitol. 2012;42(3):287-93

5. Wang L, Zhang H, Zhao X, Zhang L, Zhang G, Guo M, et al. Zoonotic Cryptosporidium species and Enterocytozoon bieneusi genotypes in HIVpositive patients on antiretroviral therapy. J Clin Microbiol. 2013;51(2): 557-63.

6. Deng L, Li W, Yu X, Gong C, Liu X, Zhong Z, et al. First report of the humanpathogenic Enterocytozoon bieneusi from red-bellied tree squirrels (Callosciurus erythraeus) in Sichuan, China. PLoS One. 2016;11(9):e0163605.

7. Inacio J, Karim MR, Dong H, Li T, Yu F, Li D, et al. Predomination and new genotypes of Enterocytozoon bieneusi in captive nonhuman Primates in zoos in China: high genetic diversity and zoonotic significance. PLoS One. 2015; 10(2):e0117991.

8. Yang Y, Lin Y, Li Q, Zhang S, Tao W, Wan Q, et al. Widespread presence of human-pathogenic Enterocytozoon bieneusi genotype D in farmed foxes (Vulpes vulpes) and raccoon dogs (Nyctereutes procyonoides) in China: first identification and zoonotic concern. Parasitol Res. 2015;114(11):4341-8.

9. Du SZ, Zhao GH, Shao JF, Fang YQ, Tian GR, Zhang LX, et al. Cryptosporidium spp., Giardia intestinalis, and Enterocytozoon bieneusi in captive non-human Primates in Qinling Mountains. Kor J Parasitol. 2015; 53(4):395-402.

10. Li W, Deng L, Yu X, Zhong Z, Wang Q, Liu X, et al. Multilocus genotypes and broad host-range of Enterocytozoon bieneusi in captive wildlife at zoological gardens in China. Parasit Vectors. 2016;9(1):395.

11. Zhang XX, Cong W, Lou ZL, Ma JG, Zheng WB, Yao QX, et al. Prevalence, risk factors and multilocus genotyping of Enterocytozoon bieneusi in farmed foxes (Vulpes lagopus), northern China. Parasit Vectors. 2016;9:72.

12. Jiang $Y$, Tao W, Wan $Q$, Li Q, Yang Y, Lin Y, et al. Zoonotic and potentially host-adapted Enterocytozoon bieneusi genotypes in sheep and cattle in Northeast China and an increasing concern about the zoonotic importance of previously considered ruminant-adapted genotypes. Appl Environ Microbiol. 2015;81(10):3326-35.

13. Ma J, Li P, Zhao X, Xu H, Wu W, Wang Y, et al. Occurrence and molecular characterization of Cryptosporidium spp. and Enterocytozoon bieneusi in dairy cattle, beef cattle and water buffaloes in China. Vet Parasitol. 2015;207(3-4):220-7.

14. Sulaiman IM, Fayer R, Lal AA, Trout JM, Schaefer FW, Xiao L. Molecular characterization of Microsporidia indicates that wild Mammals Harbor host-adapted Enterocytozoon spp. as well as human-pathogenic Enterocytozoon bieneusi. Appl Environ Microbiol. 2003;69(8):4495-501.

15. Qi M, Luo N, Wang H, Yu F, Wang R, Huang J, Zhang L. Zoonotic Cryptosporidium spp. and Enterocytozoon bieneusi in pet chinchillas ( Chinchilla lanigera ) in China. Parasitol Inter. 2015;64(5):339-41.

16. Perec-Matysiak A, Bunkowska-Gawlik K, Kvac M, Sak B, Hildebrand J, Lesnianska K. Diversity of Enterocytozoon bieneusi genotypes among small rodents in southwestern Poland. Vet Parasitol. 2015;214(3-4):242-6.
17. Guo Y, Alderisio KA, Yang W, Cama V, Feng Y, Xiao L. Host specificity and source of Enterocytozoon bieneusi genotypes in a drinking source watershed. Appl Environ Microbiol. 2014;80(1):218-25.

18. Sak B, Kvac M, Kvetonova D, Albrecht T, Pialek J. The first report on natural Enterocytozoon bieneusi and Encephalitozoon spp. infections in wild eastEuropean house mice (Mus musculus musculus) and west-European house mice (M. m. domesticus) in a hybrid zone across the Czech RepublicGermany border. Vet Parasitol. 2011;178(3-4):246-50.

19. Danisova O, Valencakova A, Stanko M, Luptakova L, Hasajova A. First report of Enterocytozoon bieneusi and Encephalitozoon intestinalis infection of wild mice in Slovakia. Ann Agric Environ Med. 2015;22(2):251-2.

20. Xu H, Jin Y, Wu W, Li P, Wang L, Li N, et al. Genotypes of Cryptosporidium spp., Enterocytozoon bieneusi and Giardia duodenalis in dogs and cats in shanghai, China. Parasit Vectors. 2016;9:121.

21. Deng L, Li W, Zhong Z, Gong C, Liu X, Huang X, et al. Molecular characterization and multilocus genotypes of Enterocytozoon bieneusi among horses in southwestern China. Parasites Vectors. 2016;9(1)

22. Peng $X Q$, Tian $G R$, Ren $G J$, Yu ZQ, Lok JB, Zhang LX, et al. Infection rate of Giardia duodenalis, Cryptosporidium spp. and Enterocytozoon bieneusi in cashmere, dairy and meat goats in China. Infect Genet Evol. 2016;41:26-31.

23. Akinbo FO, Okaka CE, Omoregie R, Adamu H, Xiao L. Unusual Enterocytozoon bieneusi genotypes and Cryptosporidium hominis subtypes in HIV-infected patients on highly active antiretroviral therapy. Am J Trop Med Hyg. 2013;89(1):157-61.

24. Shi K, Li M, Wang X, Li J, Karim MR, Wang R, et al. Molecular survey of Enterocytozoon bieneusi in sheep and goats in China. Parasit Vectors. 2016;9:23.

25. Li J, Qi M, Chang Y, Wang R, Li T, Dong H, et al. Molecular characterization of Cryptosporidium spp., Giardia duodenalis, and Enterocytozoon bieneusi in captive wildlife at Zhengzhou zoo, China. J Eukaryot Microbiol. 2015;62(6):833-9.

26. Liu W, Nie C, Zhang L, Wang R, Liu A, Zhao W, et al. First detection and genotyping of Enterocytozoon bieneusi in reindeers (Rangifer tarandus): a zoonotic potential of ITS genotypes. Parasit Vectors. 2015;8:526.

27. Li W, Li Y, Song M, Lu Y, Yang J, Tao W, et al. Prevalence and genetic characteristics of Cryptosporidium, Enterocytozoon bieneusi and Giardia duodenalis in cats and dogs in Heilongjiang province, China. Vet Parasitol. 2015;208(3-4):125-34.

28. Roellig DM, Salzer JS, Carroll DS, Ritter JM, Drew C, Gallardo-Romero N, et al. Identification of Giardia duodenalis and Enterocytozoon bieneusi in an epizoological investigation of a laboratory colony of prairie dogs, Cynomys ludovicianus. Vet Parasitol. 2015;210(1-2):91-7.

29. Mirjalali H, Mirhendi H, Meamar AR, Mohebali M, Askari Z, Mirsamadi ES, Rezaeian M. Genotyping and molecular analysis of Enterocytozoon bieneusi isolated from immunocompromised patients in Iran. Infect Genet Evol. 2015;36:244-9.

30. Li N, Xiao L, Wang L, Zhao S, Zhao X, Duan L, et al. Molecular surveillance of Cryptosporidium spp., Giardia duodenalis, and Enterocytozoon bieneusi by genotyping and subtyping parasites in wastewater. PLoS Negl Trop Dis. 2012;6(9):e1809.

31. Laatamna AE, Wagnerova P, Sak B, Kvetonova D, Xiao L, Rost M, et al. Microsporidia and Cryptosporidium in horses and donkeys in Algeria: detection of a novel Cryptosporidium hominis subtype family (IK) in a horse. Vet Parasitol. 2015;208(3-4):135-42.

\section{Ready to submit your research? Choose BMC and benefit from}

- fast, convenient online submission

- thorough peer review by experienced researchers in your field

- rapid publication on acceptance

- support for research data, including large and complex data types

- gold Open Access which fosters wider collaboration and increased citations

- maximum visibility for your research: over $100 \mathrm{M}$ website views per year

At BMC, research is always in progress.

Learn more biomedcentral.com/submissions 